\title{
Exploring the Negative Attitude in the Study of Anti-aging
}

\author{
Yingying Dai ${ }^{1, a}$ \\ ${ }^{1}$ Leonard Davis School of Gerontology, University of Southern California, CA 90033, US. \\ adaiyingyingnicole@foxmail.com
}

Keywords: Negative Attitude, Anti-aging, Aging population

\begin{abstract}
The topic of anti-aging science has been discussed widely from ancient time to nowadays. Many people are interested in anti-aging, from ancient emperors to ordinary middle aged women. Some people may argue that anti-aging is a positive attitude that they want to stay young. It is understandable if people argue that they would like to stay healthy, but, when they are discussing the methods to stay young, they may have recognized aging as an illness and have devalued aging. The very important thing that we should know is aging is natural process not an illness and we cannot avoid it.
\end{abstract}

\section{Introduction}

This paper would discuss the current studies in anti-aging science first, and discuss the natural meaning of aging then. After that, this paper would combine some cases to analyze the negative attitude in the study of anti-aging and how it affects the aging population.

\section{Current Studies in Anti-aging}

The aging population has been expanding and leads to a serious aging problem worldwide. Thus, anti-aging science has been discussed widely, and we can find many people are interested in it. Anti-aging science is self defined as a preventive science, and the current anti-aging medicine in the market usually combines nutritional recommendations, dietary supplements, prescriptions for hormones and various aesthetic techniques (Cogan, 2015). The essential goal of anti-aging science is to reduce the risk of aging, from all the psychical, physical and aesthetical aspects.

Basically, there are four types of anti-aging science, they all have different perspectives of aging and the way to anti-aging (Vincent 2006). First, the symptom alleviation. The anti-aging scientists are making efforts to cover the biological effects of aging, and they argue that the appearances are all the results from aging. There are three main categories of anti-aging medicines from this type: (1) cosmetic: it mainly refers to cream, lotion, mask, etc.; (2) supplemental pills, like vitamin pills or calcium tablet; (3) Compensatory medicine: basically, it refers to some function compensatory medicine, like Viagra (Vincent 2006). Second, life expectancy extension. In this type, anti-aging scientists argue that aging can be distinguished with youth by disease. It means the anti-aging scientists, who are in favor of this theory, related disease to aging and define disease as the symbol of aging. Third, lifespan extension. In this type, some anti-aging scientists argue that combating disease is increasing lifespan. They define old age as a failure of life. Fourth, abolition. Some anti-aging scientists in this perspective are overconfident that they argue immortality can be created. It is easy to find that some of these anti-aging science are misleading the concept of old age, and some anti-aging scientists devalue old age and regard old age as a failure.

Vincent (2006) pointed out that some recent developments have raised the possibility of extending the human lifespan. However, Cogan (2015) indicated that many anti-aging scientific studies are limited in animals or in vitro models which can not be guaranteed to have same effects in human. In addition, the leading gerontologists has declared that there was no therapy in the current market has been proved to stop or slow human aging. 


\section{Why are people interested in anti-aging?}

Anti-aging is always a popular topic: many stories are related to how difficult the ancient people were to look for the methods to stay young; some of our older parents or relatives are interested in the diet to anti-aging and some of them may even consult us about what kind of cosmetic is effective for anti-aging. Many articles and evidences have stated that anti-aging science is not mature at least at this period, however, the anti-aging science is still drawing much attentions.

With the rapid development of society, the public focuses more on the youthful appearance of people, and it can be proved from the dramatically growing market of anti-aging skin care products (Sharma \& Bowe, 2016). Some people argue that the skin with no winkles is the most beautiful skin and they assume that the beautiful skin would bring them confidence and happiness. Thus, they are willing to try any method that maybe helpful for keeping their skin stay young.

Several human diseases and illness, such as Alzheimer's, Parkinson, skin cancer and osteoporosis, would have a higher risk to be developed when age increases (Quadagno, 2012). Thus, people would easily relate aging to disease, as a result, they do not like these disease, as well as aging.

People have stereotypes of aging people, and some of these stereotypes are not positive. They may consider the aging people are all lonely or dependent. Some media also mislead condition of the later years' life to younger generations sometimes, they made publicities to the younger generation and ask them to give more concerns to the aging people. It is a good phenomenon to call more attentions for aging people, however, many aging people have a happy later years' life, and some younger generations may consider later years' life with few concerns and negative mood is a normal situation. Thus, some younger people may have emotions with rejection to aging (Schoemann \& Branscombe, 2011).

In addition, some people relate aging to death, and they do not want to face the truth that they would die in the end. A few people are extremely wealthy, and very a few may be lack of science knowledge that they would like to use money to solve all the problems, including the method to be an immoral.

\section{The Meaning of Aging}

The maximum human lifespan is about 120 years, and no valid records of humans living beyond that. It means death is a natural process of human. People cannot avoid it and they should face this truth and keep a positive attitude to it.

The biological aging process may not be the result of a rigid genetic program, however, aging cannot be recognized as a disease. Some people may get disease or some illnesses when they get old, however, it does not mean when people get old, they would develop these diseases or illness certainly. The wear and tear theory indicates that joints and bones become damaged over time as the result of age increase (Quadagno, 2012). The somatic mutation theory also states that cells of human body are damaged by radiation, mutate, or experience genetic changes, and many body functions decline over time during one' s later years' life.

Aging has the significant meaning to both old people themselves and the society. To aging people, they can enjoy their leisure later years' life to do some supplant work. They can participate in some meaningful activities or volunteer in some non-profit organizations. Furthermore, aging people can realize their own dreams in their later years' life, like spending time in their interests. Old people are not all alike. For example, bingo is not the only activity that old people would like to participate in. To aging people, there are many other activities they are interested in, like fitness, dancing, painting, directing, etc. Some old people even made great achievement in these activities. The later year' s life of old people can be various and full of meaning.

To the society, old people are important generation of the whole society, and many of them contributes a lot to the society. First, old people overall have patriotism, bravery, family values and altruism. The positive characters of old people are worthy to be learned by the younger generations. Second, old people have more experiences in both workplace and daily life. To the newbies in 
workplace, older people can always tell and teach them the principles in the work. In daily life, our older grandparents always tell us the taboos, like what kind of behaviors are rude.

Aging people are valuable group of population in our society, and aging is significant to old people themselves. Aging is natural and meaningful (Baars, 2013).

\section{Ageism in Anti-Aging Science}

Some people are in favor of anti-ageing science, however, some people are not. The main reason that lead them have different views is that they have different attitude towards aging. People who are in favor of anti-aging science, usually recognize aging as a failure, and try to avoid it. In the other side, people who are not in favor of anti-aging science have a positive attitude towards aging, and they have realized that aging is a natural process of human' s lifespan that we cannot avoid it. People who recognize aging as a failure can be considered as they have negative attitudes to aging and they have ageism in varying degrees.

The term ageism was first coined in 1969 by Robert Neil Butler who was the first director of the National Institute on Aging (Palmore, 1999). Ageism basically refers two types of behaviors which are discrimination and prejudice (Quadagno, 2012). When people developed ageist attitudes, they do not consider older people as individuals, but make judgments to old people as they are just one category of the society.

Ageism is a common problem, in a survey, more than half of aging participators have experienced ageism (Quadagno, 2012). It is also reflected in anti-aging science field. Some of the anti-aging scientists and people who are addicted in anti-aging science have different degrees of ageism.

When people are cared about the appearance of aging people and try to keep younger looks, they may have already consider the biological aging appearance as ugly. It is not a bad thing that people, especially females, like to keep a beautiful appearance. However, winkles and some age spots can not be avoided with age increase. Some new clinic beauty technologies are able to remove winkles and age spots (Pappas, 2014). It is an exciting technology to people who are care about their appearance. However, we cannot admit that the appearance is only factor of aging, and it is only one aspect of biological aging. Obviously, biological aging can not cover all aspects of aging. When some anti-aging scientist are trying to hide the appearance of aging and call it anti-aging, they have misunderstood the meaning of aging and recognize aging appearance as not beautiful (Hurd, 2011). In addition, some supplemental pills are stated by some anti-aging scientists to maintain the health condition (Mykytyn, 2006). Supplemental pills usually do not have the same affections as the instructions stated. However, due to some people have a fear to aging, they would be gullible sometimes. In some other conditions, some people just want to spend some money or energy to anti-aging to comfort themselves. Thus, we can find that some people have a potential consciousness to anti-aging. The ageism has been deeply ingrained in their mind.

Aging can not be distinguished with youth only by disease status. Disease status is not the symbol of aging, and it does not mean when you aged, you would get diseases or illnesses. Some age-related diseases or illnesses is only related to aging, but aging is not the only cause of these diseases or illnesses. Diseases develop with many reasons, such as genes, environmental conditions, personal life behaviors, etc. For example, Alzheimer' s disease, we all know it as an age related disease. However, the main causes of Alzheimer' $s$ are various. The cause of Alzheimer's disease is genes usually involved as one of the main causes. The other causes of Alzheimer' s include: a history of head injuries, depression or hypertension. Thus, we can find that aging is not the cause of diseases, it can be just described as related to some certain diseases. Some people do not need to worry about aging just because they are afraid of getting diseases. We can do more exercise, pay more attentions to the diet and environment, develop more habits which are benefit to our health. However, anti-aging is not equal to prevent diseases.

In another perspective, combating diseases is not anti-aging. As we analyzed above, aging is not equal to aging. When you get a disease, it does not mean you are aging. In another word, when you 
have beaten a disease and your lifespan might be increase after that, but you are not meant to succeed in anti-aging. Some people may have this idea is also because they recognize aging as a disease.

Some people are addicted in being immortals. They devalue aging and recognize aging as a failure in human' s lifespan. Aging and dying are all natural processes, and they are significant processes to make the life circle works. Aging is not a failure, and aging has its own meanings. We should show our respects to the natural process and life circle.

\section{The Causes of Ageism and The Methods to Prevent Ageism in Anti-Aging Science}

Ageism is produced by many aspects. Some people might have senior family members who suffers from serious disease, and they do not like to go through those painful period of time. Those people may consider older people in their family as a heavy burden to them. However, they have not realized that if they give more support and more concerns to old people, the pain of old people may release, at least in the psychological aspect.

Some people might be very cared about their appearance, such as they do not want winkle to appear in their face. First, they should be aware of that the aging appearance is only one part of aging. Keeping young appearance does not mean anti-aging. Second, they should change their attitude to aging appearance. Winkles and aging spots are not as ugly as some people thought. Winkles are the experience lines in one' s lifespan. Third, some people should build confidence in their inner beauty. It is not reliable to depend confidence and happiness on the appearance.

To prevent the ageism in anti-aging science, several advices can be taken into consider:

First, more educations about life process. Sometime, ageism appears because of people are lack of the knowledge about life process. Younger generations should be given more lectures about lifespan. Therefore, they would realize that aging and death are the natural processes.

Second, more publicities about the happy later years' life. Some people may have negative stereotype about later years' life, and they assume later years' life is lonely, dependent and boring (Nelson, 2002). It shows the public do not have enough reports about the happy and meaningful later years' life of some old people. In addition, if people can make a specific life plan and economy plan, they would adapt the later years' life easily and more likely to live in a happy old age.

Third, more educations about the relationship between disease and aging. "Aging is not a disease" . It should be spread to the public. Furthermore, if people have more knowledge about the causes of diseases, they may release the rejection emotion to aging.

\section{Conclusion}

The current anti-aging science in the market has not been proved to have the effects to stop or slow aging. After analyzing the basic types of anti-aging science, we find that anti-aging science are attempting to hide the appearance of biological aging, to mislead the concept of aging and to confuse aging with disease. Aging is a natural process and we can not avoid it. Aging is meaningful, and aging life could be various and happy.

Some people are lack of the knowledge about aging and disease, and they maybe too dependent on their appearance. Thus, they would have negative attitude to aging and be addicted in anti-aging. If people would understand more specifically about aging, their ageism may decrease. Although we can not avoid aging, we can make effort to live a healthy, energetic and meaningful old age. Moreover, a positive attitude to aging is a significant factor to face aging and enjoy a happy later years’ life. 


\section{References}

[1] Shenkman G, Ifrah K, Shmotkin D. The association between negative attitudes toward aging and mental health among middle-aged and older gay and heterosexual men in Israel[J]. Aging \& Mental Health, 2017:1.

[2] Lier A V, Tostmann A, Harmsen I A, et al. Negative attitude and low intention to vaccinate universally against varicella among public health professionals and parents in the Netherlands: two internet surveys[J]. BMC Infectious Diseases, 2016, 16(1):1-12.

[3] Heberlein T A, Ericsson G. Public attitudes and the future of wolves Canis lupus in Sweden[J]. Wildlife Biology, 2016, 14(Sep 2008):391-394.

[4] Ramjan L, Hunt L, Salamonson Y. Predictors of negative attitudes toward Indigenous Australians and a unit of study among undergraduate nursing students: A mixed-methods study[J]. Nurse Education in Practice, 2015, 17:200-207.

[5] Paik S. Perception of grandchild-grandparent communication in South Korea and reduction of prejudice toward aging[J]. European Child \& Adolescent Psychiatry, 2014, 18(4):240-249.

[6] Challapallisri V, Dempster L V. Attitude of doctors towards mentally ill in Hyderabad, India: Results of a prospective survey[J]. Indian Journal of Psychiatry, 2015, 57(2):190.

[7] Lavee J, Fiatarone S M, Trey T, et al. The uninvestigated factor behind the negative attitudes toward cadaveric organ donation in China.[J]. Transplantation, 2014, 98(8):78-9.

[8] Popescu C A, Buzoianu A D, Suciu S, et al. The impact of antistigma education on the attitudes of first-year medical students towards mental illness[J]. European Psychiatry, 2016, 33:S542-S542.

[9] Hussain Z, Yusoff Z M, Sulaiman S A. A study exploring the association of attitude and treatment satisfaction with glycaemic level among gestational diabetes mellitus patients.[J]. Primary Care Diabetes, 2014, 9(4):275-282.

[10] Cataldo J K, Hunter M, Petersen A B, et al. Positive and instructive anti-smoking messages speak to older smokers: a focus group study[J]. Tobacco Induced Diseases, 2015, 13(1):1-8. 